\title{
Community-centric engagement: lessons learned from privacy awareness intervention design
}

\author{
Lizzie Coles-Kemp \\ Information Security Group \\ Royal Holloway \\ Egham TW20 0EX \\ lizzie.coles-kemp@rhul.ac.uk
}

\author{
Debi Ashenden \\ Informatics and Systems Engineering \\ Cranfield University \\ Shrivenham SN6 8LA \\ d.m.ashenden@cranfield.ac.uk
}

\begin{abstract}
Visualisation and Other Methods of Expression (VOME) was a research project with the main objective of developing methods of expressing a wide range of on-line privacy-related concepts. Having built this wider vocabulary, VOME was also tasked with developing a range of privacy awareness interventions for both on and off-line contexts. Examples of VOME interventions include a card trading game, participatory video and embedded on-line interaction tools. In order to develop these interventions, VOME conducted a range of user-centric and participatory design engagements in communities across the UK. Engagements included: think-out-loud technology evaluations, participatory video development, "show and tell" family workshops, participatory theatre and community art collage building. Within each of these engagements, the project used qualitative social research to ground the design of its interventions. The communities in which VOME engaged represented a broad range of social and economic demographics and demonstrated varying levels of digital literacy. During its three and half years of engagement, VOME built up a rich set of lessons learned from using such a wide range of engagement approaches. The lessons learned address a number of key topics: tension of engagement control between participants and researchers, importance of appropriately situating the engagement and limitation of participant segmentation. This short paper outlines VOME's key findings within each of these topics.
\end{abstract}

Key words: Engagement, intervention, digital literacy, privacy awareness.

\section{INTRODUCTION}

Visualisation and Other Methods of Expression (VOME) is an interdisciplinary project that focuses on questions related to privacy, identity and consent in on-line services. The research is used to ground the development of technology designed to support privacy and consent decision-making. Examples of VOME interventions include a card trading game, participatory video and embedded on-line interaction tools, for example interactive information flow maps. From the start, VOME set out to engage with citizens who, to date, had not been included in privacy design studies. Sunderland City Council gives the example of one such group of citizens:

"Consider the case of a young person, aged 13 years, who is at risk of offending. They are one of the target groups for the Empowering Young People programme which will use on-line service delivery as part of the method of delivery. Some of their peers have heard about the scheme and are saying it will be used by the police to keep track of them. Our young person is shy, reserved and has some learning difficulties. How will they express their concerns about how their data will be used, or will they simply choose not to engage? How might this be further complicated if the young person were to be a member of a minority ethnic group? Would a set of tools designed for youth work support workers help clarify the issue, and engage the young person? How might the youngster explain how the scheme safeguards information rights to their peers?" Conn Crawford, Sunderland City Council.

The project teams conducted a number of qualitative social research studies that required researchers to explore how participants engage with technology and the decision-making logic in operation when personal information is disclosed. 
The research teams realised quite quickly that traditional qualitative methods had limitations with the groups with which the project wanted to engage. In particular, researchers learned that unintentionally the traditional data gathering and engagement approaches were not inclusive and partially or completely excluded those with lower levels of digital and visual literacy and those with lower levels of social confidence. For example, our mixed method studies which used light technological evaluation, observations, interviews and surveys also proved challenging for those with lower levels of digital and visual literacy (ColesKemp and Zabihi, 2011). This realisation both influenced the research approaches taken and method and media for data gathering.

\section{APPROACH}

In VOME, the traditional approaches found in security and privacy technology design were supplemented with more inclusive data gathering approaches. These approaches include: think-outloud technology evaluations, participatory video development, "show and tell" family workshops, participatory theatre and community art collage building. Using these approaches also taught the researchers about the need to cede control to participants and carefully segment communities into different constituencies. These approaches also clearly showed the need to design culturally sympathetic research approaches and to carefully select the media for data collection.

\subsection{Participatory Video}

VOME supported Hudson's Youth Project in the London borough of Newham to produce two videos - one a documentary video that the VOME project specifically commissioned and the other a music video that the young people asked if they could make. The aim was to create a participatory video where the young people determined what was filmed, how it was produced and, most importantly, the message conveyed. With the documentary video researchers were part of the focus groups that were run to stimulate discussion and the ensuing video displays strong characteristics of researcher involvement. The music video on the other hand required no input at all from the researchers and used a medium that the young people were more comfortable with. The music video, 'Internet Saint, or Online Demon?', was a more effective approach for gathering young people's views as it was made without intervention from researchers. This result showed that the design approach needs to use a medium for data gathering that is culturally sympathetic to the target demographic.

\subsection{Think-Out-Loud Technology Evaluations}

VOME used think-out-loud technology evaluations to better understand the logic behind personal information disclosure in different situations. For example, researchers developed an on-line registration prototype which represents a mock-up council, named Your Local Council (YLC), which offers an on-line smartcard registration service. This is a situation that many of the participants were familiar with and was situated in a relationship with an institution that many of them had. In the research activity, after the introduction to smartcard services provided by YLC, users were asked to interact with the website which guides them through a sequential registration process that consisted of the following pages:

(i) Introduction to smartcard services and selection of services - The user starts by selecting one or more services.

(ii) About us

(iii) Personal Information Requirement - The service provider informs the user that certain personal information is required to use the service, what will happen to the data as well as the reason for collecting it. This information will be communicated to the user with help of an interactive dataflow diagram that displays who has access to what type of personal information.

(iv) Service Agreement - The service provider gives a contract of their agreement with the user to keep as a reference. It is an overview of selected services, privacy policy and terms \& condition.

(v) Registration - The final step of the process is a registration page that displays a form where the user discloses the necessary personal information for acquiring the selected service(s).

Participants were exposed to three privacy interventions during the registration process. Privacy awareness was measured using a mixedmethods approach in three parts:

- Questionnaire to categorise the participants in terms of their digital literacy.

- Engagement with digital probes (YLC website) to encourage reflection (captured through think-out-loud)

- Interview to evaluate levels of privacy awareness with the participant.

\section{3 "Show and Tell” Family Workshops}

One drawback to the think-out-loud technology evaluation in the previous section is that it constrained participants to reflect on a particular type of technology in a particular context. 
As a result, VOME ran a number of "show and tell" workshops where a small group of participants, matching a particular set of criteria, would spend time showing and reflecting how they used ICT within the family setting and talking about the type of personal information that they disclosed in the process. In this approach, in contrast to the thinkout-loud technology evaluations, participants situate their technology use in a context to which they can relate. Participants also shape the flow of the engagement by bringing up points for discussion from the reflection sessions.

One example of such a workshop took place in northern England in July 2011. The workshop involved six granddaughters (GDs) and six grandmothers (GMs). It was staged in the part of a northern English town where local granddaughter/grandmother pairs could be recruited through a community center. Preliminary work had already identified that there were close family pairings and internet active family members who met the criteria for participation.

The GMs were aged 55+ and four of the six GMs were great-grandmothers. They included a mix of active social networkers and those without accounts for any social networks. One GM used social networking to keep contact with relatives in Australia. Two of the others used the Internet for email and on-line shopping. The non-social networkers all had experience of family members who use social networks. The event was facilitated by a community leader and a VOME researcher. The workshop began with an introduction to VOME's research and the process for the day. This explained that VOME's work is on personal information control, but didn't develop the theme. The "show and tell" session in this instance used GMs and GDs in a pair. Each GD/GM pair used the big screen and the computer in the workshop room to show their activities. The participant group was then encouraged to discuss issues that arose and write down thoughts and reflections. The follow-on "show and tell" session picked up on the themes identified in the reflection session.

\subsection{Participatory Theatre and Community Art}

Whilst "show and tell" family workshops moved VOME researchers some way towards participantled situated research, researcher intervention was still present and for some participants that presence was a barrier to engagement with the research. As a result, VOME pushed further towards participant-led research engagements by using participatory theatre and community collage making. This form of engagement would often involve collaboration with performance artists, in particular clowns, who initiate engagement with members of the public and introduce them to some very simple research tasks.

One example of this is collage building in Middlesbrough Railway Station. The focus of this activity was to ask members of the public what type of secrets they keep on-line and what secrets they try to find out on-line. Performance artists initially engaged with participants. Those who then wanted to answer research questions were invited to produce some drawings, text or sound recordings for a collage that was built in the underpass of Middlesbrough railway station. The same approach was used across four community centres, the central library and the city museum in Sunderland. This engagement approach had the least direct input from researchers in terms of participant engagement but had considerable input from the researchers in the setup, observational work while the engagement was on-going and post engagement analysis.

\section{LESSONS LEARNED}

Lessons learned from developing the engagement programme can be grouped into three areas: control tension, situation and segmentation limitation.

\subsection{Control Tension}

When designing engagements, a tension emerged between the control the researcher needed to have over the engagement and the control that the researcher needed to cede to the participants.

During the initial engagements such as the participatory video and think-out-loud technology evaluations, the need to give control to participants (Ospina et al, 2008) became clear. This means that engagement cannot start from a fixed question, but that researchers have to interpret what is produced during the engagement. Researchers also learned that even light control might be too dominant for some groups of participants. Engagement with communities is often mediated through powerful stakeholders such as community and youth workers. Therefore, in terms of control, it is also important to understand the involvement and influence of stakeholders close to the end users. This is often unavoidable but, as French et al (2010) point out, such stakeholder influence needs to be acknowledged in any engagement activity as it will have an impact on how messages are received.

The documentary video made by Hudsons Youth Project demonstrated too much control by researchers as researchers' words were repeated back through the video suggesting that what was presented was what the young people believed the 
researchers wanted to hear rather than what they actually felt.

In order to cede control to participants, design researchers need to reduce the influence of researchers and gatekeepers as far as possible and select media for data collection that is culturally sympathetic to the target demographic.

\subsection{Situation}

Engagement fails when it assumes a transmission model of communication, ignores lay or public perceptions and when it presents technological fait d'accompli. The need to develop insight is emphasised by French et al (2011) and the key lesson to take away is that for engagement to be successful it is vital to understand end users and the contexts that determine their attitudes and behaviours from their point of view - not how the researcher imagines it to be.

In the think-out-loud technology evaluation, the context of registering with an on-line Council service did not resonate with all the participants. Also, those evaluations were run in a community or UK online centre and this was not a physical space that all the participants would use for on-line registration. Another situational issue was the lack of family context. Many of the less digitally capable participants would typically register for on-line services with the help of family members. In this case, on-line engagement without other family members present as well as outside the home was not natural. As a result, fewer participants with this type of background took part in the think-out-loud technology evaluation and for those that did; their evaluation was stilted because of their lack of familiarity with the context.

Therefore, design researchers need to recognise the importance of appropriately situating the engagement in as natural a setting as possible. This is often referred to as 'ecological validity' (Hayes, 2000, p.105) and is often absent in laboratory-based studies.

\subsection{Limitations of Segmentation}

As was seen with the participatory video, the manner in which an engagement is responded to depends on the segmentation of the participants. Also, there will be a range of responses within each segment. In particular, issues of digital literacy, degree of stability in home and social lives, cognitive capacity and degree of social confidence were some of the dominant factors in influencing segmentation response. It is therefore important that design researchers do not present the experiences of demographic segments as a homogeneous group with a single consistent attitude towards technology and risk. Otherwise the research stands to unknowingly alienate an often significant proportion of the user community. More subtle approaches to segmentation are needed. It is also important for researchers to recognise that different research focuses need different types of segmentation. In the VOME project, we found that community-based segmentation had an important role to play (Andreason, 2006) and therefore segmentation according to family settings was important.

\section{CONCLUSION}

The push towards participant- controlled engagement was a valuable journey. As intervention designers, we were able to gather the views and inputs of a wider audience. By not taking account of the lessons learned systems are likely to be designed that end users will resist using either because they don't take sufficient account of existing attitudes and behaviours or because they do not accord with how systems are used in a realworld setting. The lessons learned will influence the methodologies used in future studies around not just privacy awareness but online awareness and behaviour in general.

\section{REFERENCES}

Andreason, A. (2006) Social Marketing in the $21^{\text {st }}$ Century. Sage, London, UK

Coles-Kemp, L., Kani-Zabihi, E. (2011) Practice Makes Perfect: Motivating confident on-line privacy protection practices. IEEE International on Social Computing, 9 - 11 October, Anonymous IEEE, pp. 866-872

French, J., Merritt, R., Reynolds, L. (2011) Social Marketing Casebook. Sage, London, UK

Hayes, N. (2000) Doing Psychological Research Open University Press, Maidenhead, UK

Ospina, S., Dodge, J., Foldy, E., Hofmann-Pimilla, A. (2008) Taking the Action Turn: Lessons from Bringing Participation to Qualitative Research. In Reason, P., Bradbury, H. (eds), The Sage Handbook of Action Research: Participative Inquiry in Action. Sage, London, UK

Reynolds, L., Merritt, R. (2010) Scoping. In French, J., Blair-Stevens, C., McVey, D., Merritt, R. (eds), Social Marketing in Public Health. OUP, Oxford, UK 